\title{
Study of seeding mechanisms of TMI with spatially and temporally resolved imaging
}

\author{
Christensen, Simon L.; Johansen, Mette M.; Michieletto, Mattia; Triches, Marco; Maack, Martin D.; \\ Lægsgaard, Jesper
}

\section{Published in:}

Proceedings of SPIE

Link to article, DOI:

$10.1117 / 12.2588432$

Publication date:

2021

Document Version

Publisher's PDF, also known as Version of record

Link back to DTU Orbit

Citation (APA):

Christensen, S. L., Johansen, M. M., Michieletto, M., Triches, M., Maack, M. D., \& Lægsgaard, J. (2021). Study of seeding mechanisms of TMI with spatially and temporally resolved imaging. In M. N. Zervas, \& C. JaureguiMisas (Eds.), Proceedings of SPIE (Vol. 11665). [116650Y] SPIE - International Society for Optical Engineering. Proceedings of SPIE - The International Society for Optical Engineering Vol. 11665 https://doi.org/10.1117/12.2588432

\section{General rights}

Copyright and moral rights for the publications made accessible in the public portal are retained by the authors and/or other copyright owners and it is a condition of accessing publications that users recognise and abide by the legal requirements associated with these rights.

- Users may download and print one copy of any publication from the public portal for the purpose of private study or research.

- You may not further distribute the material or use it for any profit-making activity or commercial gain

- You may freely distribute the URL identifying the publication in the public portal 


\section{Study of seeding mechanisms of TMI with spatially and temporally resolved imaging}

Christensen, Simon, Johansen, Mette, Michieletto, Mattia, Triches, Marco, Maack, Martin, et al.

Simon L. Christensen, Mette M. Johansen, Mattia Michieletto, Marco Triches, Martin D. Maack, Jesper Lægsgaard, "Study of seeding mechanisms of TMI with spatially and temporally resolved imaging," Proc. SPIE 11665, Fiber Lasers XVIII: Technology and Systems, 116650Y (5 March 2021); doi: $10.1117 / 12.2588432$

SPIE. Event: SPIE LASE, 2021, Online Only 


\title{
Study of seeding mechanisms of TMI with spatially and temporally resolved imaging
}

\author{
Simon L. Christensen ${ }^{\mathrm{a}, \mathrm{b}}$, Mette M. Johansen ${ }^{\mathrm{b}}$, Mattia Michieletto ${ }^{\mathrm{b}}$, Marco Triches $^{\mathrm{b}}$, Martin \\ D. Maack ${ }^{\mathrm{b}}$, and Jesper Lægsgaard ${ }^{\mathrm{a}}$ \\ ${ }^{a}$ DTU Fotonik, Technical University of Denmark, Ørsteds Plads, DK-2800 Kgs. Lyngby, \\ Denmark

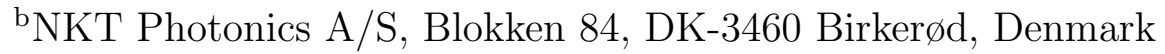

\begin{abstract}
In this work we investigate transverse mode instability (TMI) in the presence of pump intensity noise and a controlled perturbation of the input coupling for a rod-type fiber amplifier using spatially and temporally resolved imaging (ST). We show that inherent pump intensity noise from the power supply can define significant peaks in the resulting TMI spectrum. ST measurements in the transition region of TMI also indicates that the simple picture of TMI being seeded by the combination of a static initial fraction of LP11 and pump or signal intensity noise is not valid for our measurements. Furthermore, we present seeding of TMI by perturbing the input coupling dynamically which allows measurements of the TMI gain as a function of frequency and signal power.
\end{abstract}

Keywords: Transverse mode instability, Beam fluctuation measurement, High power, Thermal Rayleigh scattering, High-speed camera, Large-mode-area fiber, spatially and temporally resolved imaging, ST measurements

\section{INTRODUCTION}

Transverse mode instability (TMI) is the main limitation in average power scaling of fiber lasers and fiber amplifiers. ${ }^{1}$ Understanding and mitigating TMI is as a consequence an important field of research and the focus of this work. It is around 10 years ago these mode instabilities were first reported ${ }^{2}$ and it is now wellestablished that the thermo-optic effect causes the amplification of TMI but seeding and resulting dynamics are still discussed. This uncertainty also expresses itself in the many models currently used to model TMI. ${ }^{3-7}$ In order to further understand TMI, detailed experimental characterizations of the phenomenon are needed. ${ }^{8}$

In our recent work, ${ }^{9,10}$ we proposed and applied the spatially and temporally resolved imaging (ST) technique which is also the basis of this work. The ST method makes use of the periodicity of TMI fluctuations and can fully describe different beam dynamics. The spatial information of specific fluctuations combined with a high sensitivity allow for detailed analysis of TMI in the transition region. This article is organized into four sections beyond the introduction. The spatially and temporally resolved imaging technique is described in section 2 . In section 3 inherent pump intensity noise of two different amplifier systems is measured and the resulting TMI spectra are investigated. In section 4 we show seeding of TMI which is explored as a function of frequency and signal power. Section 5 summarizes the work presented in the section 3 and 4 .

\section{SPATIALLY AND TEMPORALLY RESOLVED IMAGING}

The ST method fully describes fluctuations of a beam by separating fluctuations in frequency as mentioned in the introduction..$^{9,10}$ The fundamental data required for this method is a movie of the beam intensity fluctuations

Further author information: (Send correspondence to S.L.C.)

S.L.C.: E-mail: slon@fotonik.dtu.dk

Fiber Lasers XVIII: Technology and Systems, edited by Michalis N. Zervas,

Cesar Jauregui-Misas, Proc. of SPIE Vol. 11665, 116650Y · (C) 2021 SPIE

CCC code: $0277-786 \mathrm{X} / 21 / \$ 21$, doi: $10.1117 / 12.2588432$ 
which is Fourier transformed in time. The Fourier transform results in a complex data structure depending on transverse space and frequency, defined as

$$
\tilde{I}(x, y, \nu)=\int_{0}^{T} I(x, y, t) \mathrm{e}^{-2 \pi i \nu t} \mathrm{~d} t
$$

where $T$ is the length of the movie in time and $I(x, y, t)$ is the time-dependent beam intensity profile. The complex data structure, $\tilde{I}(x, y, \nu)$, contains the spatial intensity and phase of fluctuations at different frequencies. No information has been removed in this analysis which also means that the data is still somewhat difficult to visualize and interpret. In order to understand the ST measurement we define a spatially averaged power spectral density (PSD),

$$
\tilde{P}(\nu)=\frac{1}{A_{\Omega}} \int_{\Omega} \frac{1}{T}|\tilde{I}(x, y, \nu)|^{2} \mathrm{~d} x \mathrm{~d} y,
$$

where $\Omega$ is the transverse domain of the camera and $A_{\Omega}$ is the area of the transverse camera domain. $\tilde{P}(\nu)$ is named the ST PSD and accurately compresses the information about fluctuation power as a function of frequency.

In practice a high-speed camera captures the movie as TMI fluctuations occur on the millisecond level. The maximum frequency a discrete Fourier transform can accurately resolve is half of the acquisition rate but fluctuations above this limit cause undersampling and will affect the ST measurement. The acquisition rate of the camera is sufficient for most cases of TMI when the noise floor is reach before the highest resolved frequency. We have developed the ST method for analysing TMI but this analysis can be applied on all movies.

\section{TMI IN THE PRESENCE OF PUMP INTENSITY NOISE}

Recent work by Stihler et al. ${ }^{11,12}$ has shown the large impact of pump intensity noise on the TMI threshold. In the cited work, a controlled white noise in different frequency ranges is applied on the pump intensity and the TMI threshold is measured. In this work we study the effect of inherent pump noise on the resulting TMI fluctuations using the ST method. ${ }^{9,10}$ The ST method allows for detailed analysis of beam fluctuations by utilizing the periodic nature of fluctuations and applications on TMI have already been fruitful.

Inherent pump noise can originate from different sources but is important to measure when dealing with TMI. We measure the pump noise by attenuating the pump light with dichroic mirrors and focusing the pump light onto a photodetector. The photodetector captures a time series of the power which can be Fourier Transformed to achieve a noise power spectral density (PSD) and a sketch of the setup can be seen in Fig. 1 a). The pump noise is measured for different powers and with two different pump power supplies. Each noise measurement is taken over 5 minutes. For each power supply the pump diode is used to amplify a signal in an aeroGAINROD-PM $85^{13}$ to around TMI threshold and the output signal is analysed with ST measurements. We use a CMOS camera from Basler, model aca640-750 $\mathrm{mm},{ }^{14}$ for the ST measurements. A sketch of the amplifier setup is shown in Fig. 1 b). The core of the rod is doped with Ytterbium hence the pump diode light is continuous wave with a wavelength of around $976 \mathrm{~nm}$. The seed laser has a wavelength of $1030 \mathrm{~nm}$ and is pulsed with a repetition rate of $80 \mathrm{MHz}$, pulse duration of $20 \mathrm{ps}$, and an average power of $15 \mathrm{~W}$ is used for measurements in this section. The same rod is used for the two amplifier measurements with different pump power supplies.

Figure 2 shows the spatially averaged PSD from the ST measurement for a specific signal power and the pump noise PSD. The pump intensity noise is shown for the pump power resulting in the signal power in the two plots. The ST measurements in this section are taken over 0.5 seconds compared to the 5 min of the pump noise and this difference causes the large difference between the noise floor of the ST PSD and the pump noise PSD. For power supply 1, the ST PSD is shown for a signal power of $375 \mathrm{~W}$ while the ST PSD is shown for a signal power $365 \mathrm{~W}$ for power supply 2 . The ST PSD for power supply 1 does not show strong fluctuations but has two significant peaks at $300 \mathrm{~Hz}$ and $600 \mathrm{~Hz}$. The PSD of the pump noise also shows peaks at these frequencies but has more peaks that are not seen in the ST PSD. The right plot however, shows a very strong correlation between the peaks in the ST PSD and the pump noise PSD as significant peaks are found at all frequencies of a whole number of $100 \mathrm{~Hz}$ in both PSDs. The strong correlation reveals that pump intensity noise clearly has a direct impact on the TMI fluctuations at the specific frequencies and that inherent pump noise 
a)

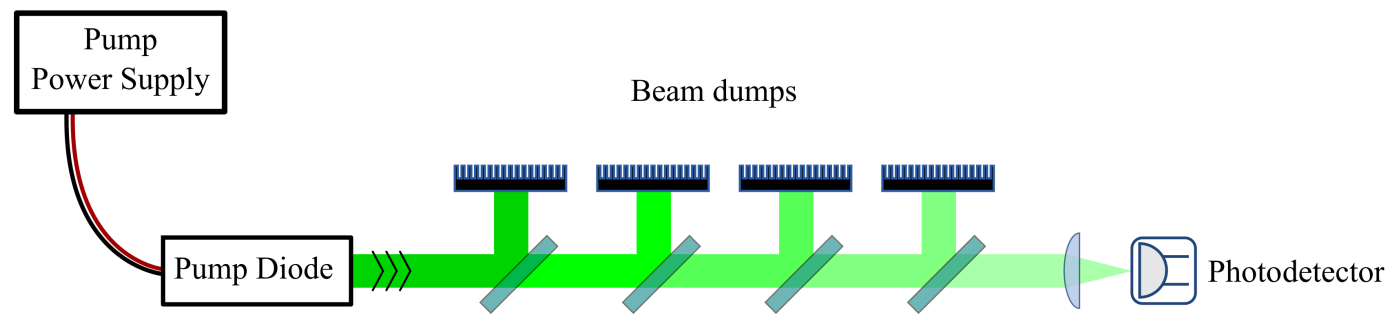

b)

Dichroic mirrors

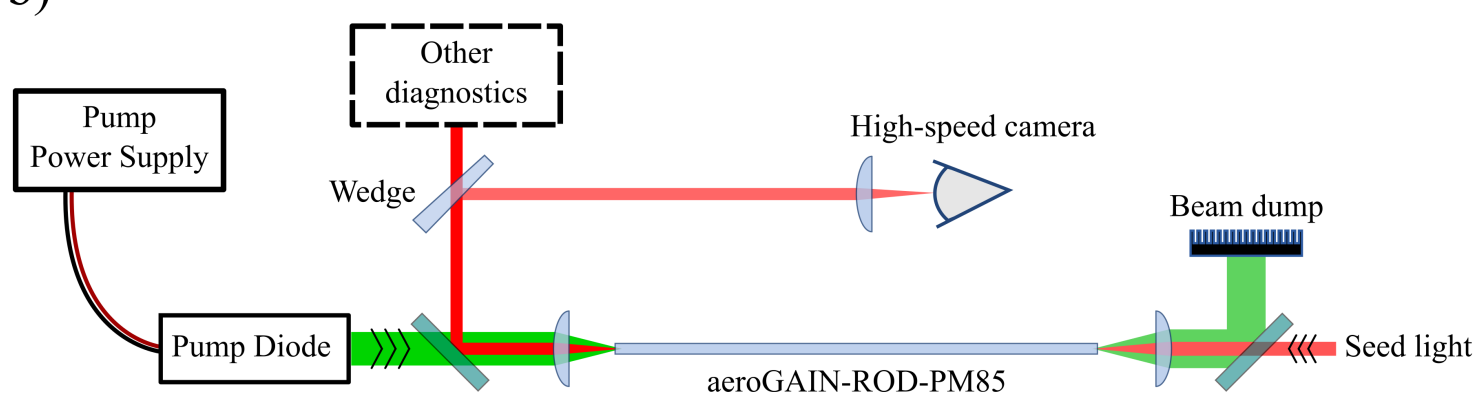

Figure 1: a) Sketch of the setup used to measure pump noise. b) Sketch of the amplifier setup.

can define the significant peaks observed in TMI. This supports the proposition by Johansen et al. ${ }^{15}$ that the significant peaks in TMI originates from electrical noise. The strength of the TMI fluctuations are stronger for
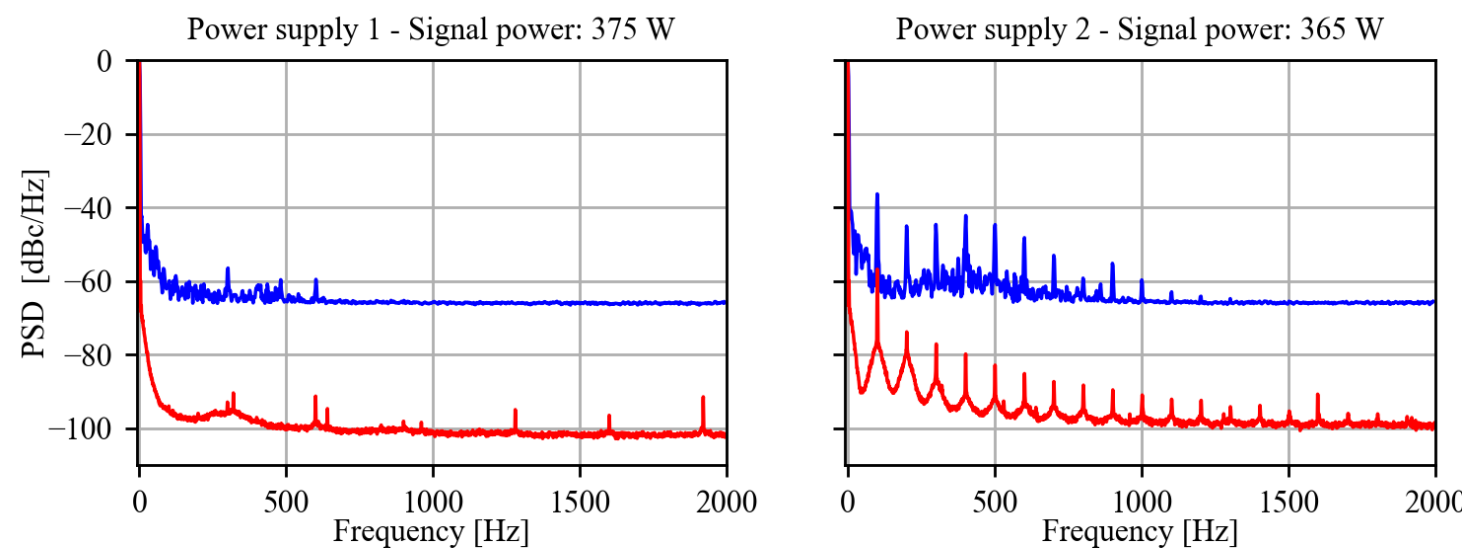

- Pump intensity noise

ST-meas. of signal

Figure 2: Plots of the PSD of the pump (red) and the averaged PSD from an ST measurement of the signal output (blue) as a function wavelength. The left plot shows the measurement when the pump diode is driven by power supply 1 and the right plot is for power supply 2 . Reprinted with permission from Christensen et al. Fig. $5^{10}$ (c) The Optical Society.

the ST measurement for power supply 2 even though the signal power is lower. A larger pump noise will lead to lower TMI thresholds but it is not possible to quantify the reduction purely through these measurements. The TMI of the pristine aeroGAIN-ROD-PM85 was first measured with power supply 1 and afterwards with power 
supply 2 and experienced an unknown amount of photodarkening between measurements.
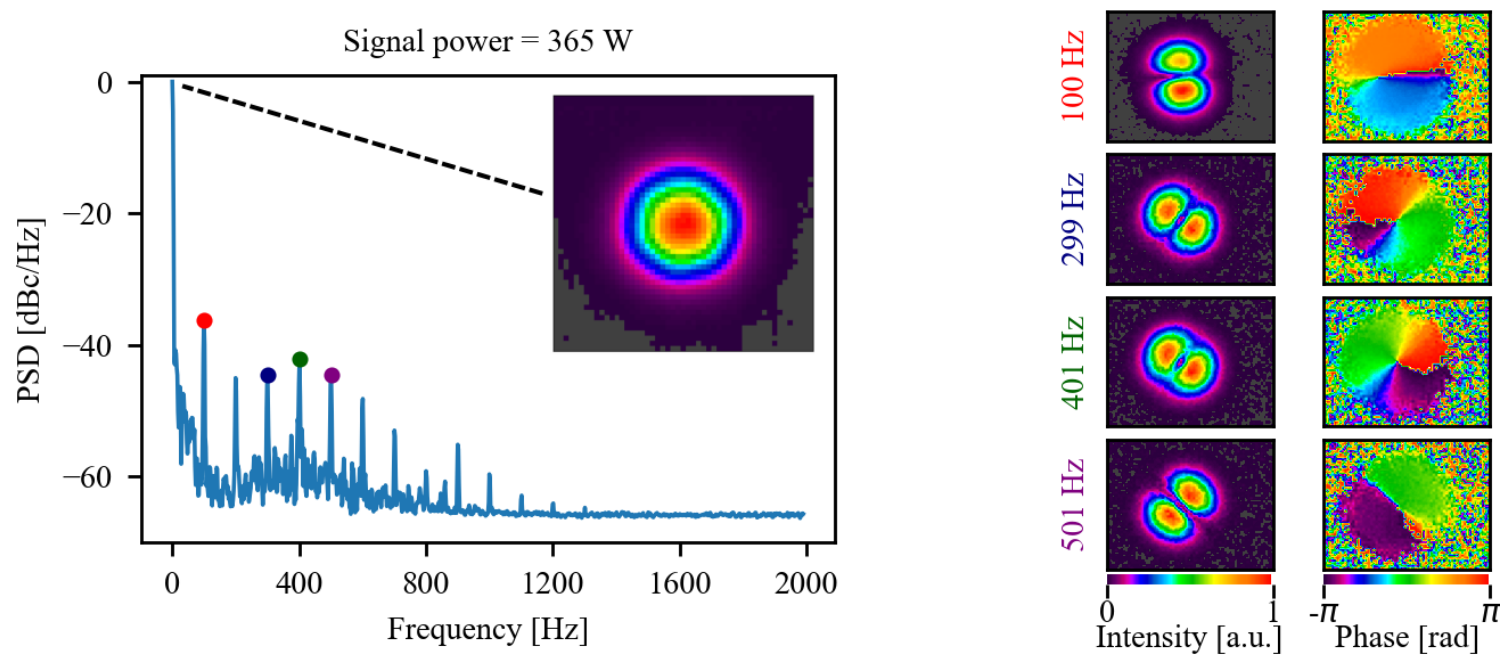

Figure 3: ST measurement of the amplifier output for a signal power of $365 \mathrm{~W}$ with power supply 2. The left plot shows the ST PSD in $\mathrm{dBc} / \mathrm{Hz}$ and has an inset of the $0 \mathrm{~Hz}$ intensity profile i.e. the mean beam profile during the movie. The spatial profiles of four different fluctuations are shown to the right and are colour coded to dots in the PSD.

Detailed analysis of beam dynamics can be performed using ST measurements as mentioned previously since fluctuations can be separated by frequency. Figure 3 displays a fraction of such an analysis on the signal dynamics for power supply 2. The spatial dependence of four fluctuations at different frequencies are defined by their intensity and phase in the transverse space. The $0 \mathrm{~Hz}$ intensity profile shows that mean beam profile is fundamental mode-like for this signal power which suggests that the TMI is in the transition region. The three fluctuations at $100 \mathrm{~Hz}, 299 \mathrm{~Hz}$, and $501 \mathrm{~Hz}$ show different rotations of the same beam dynamic which is a pure direction $\mathrm{LP}_{11}$-mode interaction. The fluctuation at $401 \mathrm{~Hz}$ is however not pure which means that the $\mathrm{LP}_{11}$-modes are out of phase. All these fluctuations are caused by the energy transfer between the $\mathrm{LP}_{01}$ and $\mathrm{LP}_{11}$-modes and are described in detail in our previous work. ${ }^{9,10}$ Many models ${ }^{3-7}$ build on the assumption that TMI is seeded by some static fraction of $\mathrm{LP}_{11}$ due to a small unavoidable misalignment combined with pump or signal noise. From this seeding mechanism the fluctuation orientation would most likely follow the orientation of the input misalignment. Some rotation could be expected due to a possible spin in the geometry fiber but this rotation would apply similarly to all fluctuations. This is clearly not the case for the TMI observed with the ST measurement which includes many different orientations and both in and out of phase $\mathrm{LP}_{11}$-modes. Consequently, the measurement indicates that assumed seeding mechanism of many models does not seed the TMI in this measurement. The pump intensity noise clearly affects the power in the TMI fluctuations and should be investigated further by introducing a controllable pump noise. TMI in different orientations is not new knowledge as Otto et al. described the temporal dynamics of TMI in the chaotic with a high speed camera and saw excitation in all orientations. ${ }^{8}$ TMI in the chaotic region is, as its name suggest, difficult to understand but the sensitivity of the ST method permits description of TMI in the transition region. In the next section a novel seeding mechanism is showed experimentally which can explain the seemingly independent fluctuations of TMI.

\section{SEEDING OF TMI WITH A KINEMATIC MIRROR MOUNT}

In this section we show that TMI in a rod fiber amplifier can be seeded by a mechanical perturbation on the seed coupling. A controlled mechanical perturbation is induced by a kinematic mirror mount with a piezo actuator ${ }^{16}$ on the input side of the amplifier. A sketch of the setup can be seen in Fig. 4. The dichroic mirror used to filter 
the residual pump light will reflect a small fraction of the seed light which is used to measure the amplitude of the induced perturbation. The lens focusing the reflected seed light into the camera is similar to the lens used for input coupling into the rod. The instability causes a harmonic translation of the input coupling which can be evaluated using the ST method. We use a few-mode Yb-doped rod which ensures that the induced instability

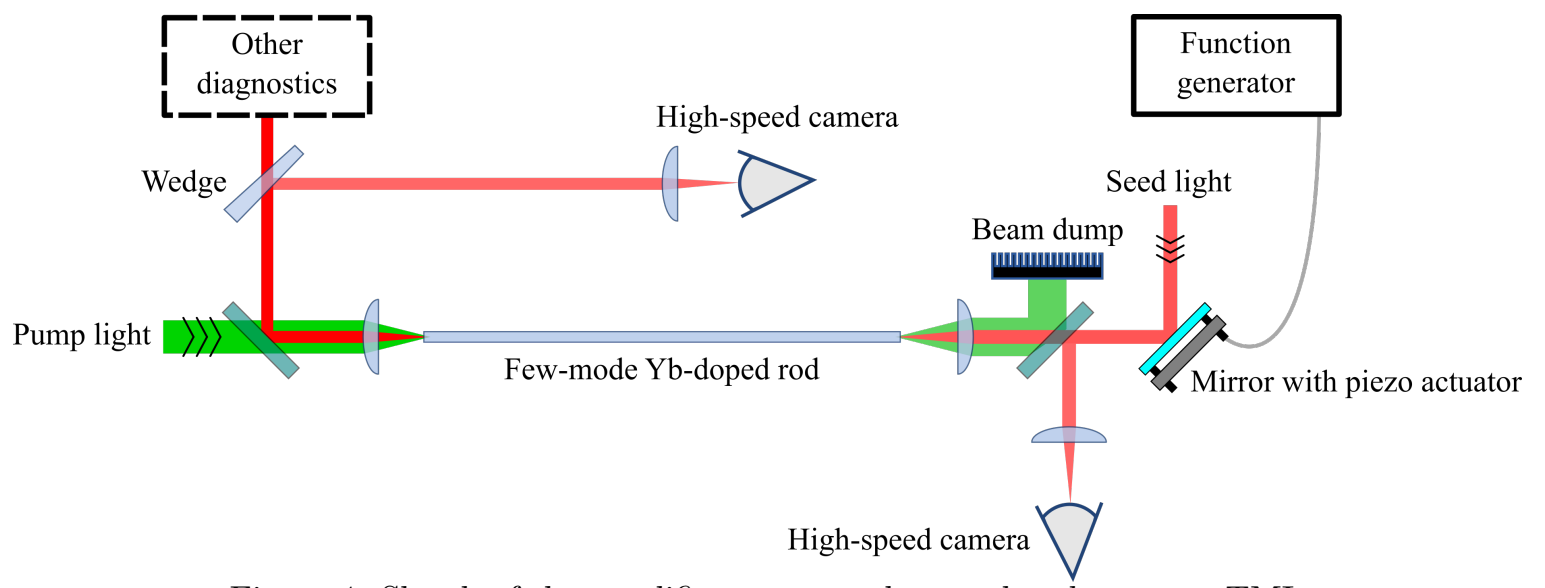

Figure 4: Sketch of the amplifier setup used to seed and measure TMI.

exceeds the noise level of the ST measurement for all signal powers. This few-mode rod is $80 \mathrm{~cm}$ long and has an MFD of $65 \mu \mathrm{m}$. For all measurements shown in this section, a seed power of $10 \mathrm{~W}$ is used and the peak-to-peak voltage of the piezo actuator is $2 \mathrm{~V}$ which roughly corresponds to an amplitude of the input beam movement of $0.16 \mu \mathrm{m}$ at the rod facet. The perturbation frequency $(\mathrm{PF})$ is the frequency of the harmonic translation in the input coupling and is variable. Power supply 1 from the previous section is used to drive the pump diode.

Figure 5 shows part of an ST measurement where the system is amplified to $180 \mathrm{~W}$ of signal power and the $\mathrm{PF}$ is $225 \mathrm{~Hz}$. Fluctuations are observed for a wide band of frequencies but at $225 \mathrm{~Hz}, 450 \mathrm{HZ}$, and $675 \mathrm{~Hz}$ very strong peaks are found. This is of course the at the PF and higher harmonics of the perturbation. With the ST measurement it is clear that these fluctuations are very different in nature. The main fluctuation at $225 \mathrm{~Hz}$ is similar to the usual TMI fluctuations as seen in Fig. 2 while each higher harmonics has an added lobe in both the intensity and phase. The spatial distribution of the peak at $450 \mathrm{~Hz}$ was discussed in our previous work ${ }^{10}$ and is due to transfer of power between $\mathrm{LP}_{01}$ and $\mathrm{LP}_{11}$-modes. The structure of the fluctuations at $675 \mathrm{~Hz}$ was however not discussed. The spatial distribution fits the development with respect to the other fluctuations but does not follow the same pattern in fluctuation strength as the peaks at $225 \mathrm{~Hz}$ and $450 \mathrm{~Hz}$. The lower power could suggest that the origin of this fluctuation differs from the two others which are directly related to TMI. This strange fluctuation is obviously connected to the main TMI fluctuations, but we believe it is an indirect response of the fiber to the main fluctuation. At the heart of TMI lies the thermo-optic interaction where heating inside the fiber leads to changes in the refractive index. The strong seeding amplified in this system leads to a strong well-defined perturbed electrical field and heat profile in the fiber. This dynamic heat change will influence the eigenmodes of the fiber driving them towards the dynamic electrical field. These dynamic changes to the eigenmode will follow the harmonics of the PF and can explain the fluctuation we observe at 675 $\mathrm{Hz}$.

Controllable seeding of TMI is very useful with respect to evaluating the effect on TMI of different variables. Figure 6 shows the TMI response as a function of frequency and as a function of signal power. To evaluate the strength of the TMI fluctuation we employ an RMS value of the main fluctuation located at the PF. The RMS value is defined as

$$
\sigma_{P F}=\sqrt{\int_{\nu_{p}-\Delta \nu / 2}^{\nu_{p}+\Delta \nu / 2} \tilde{P}(\nu) \mathrm{d} \nu},
$$



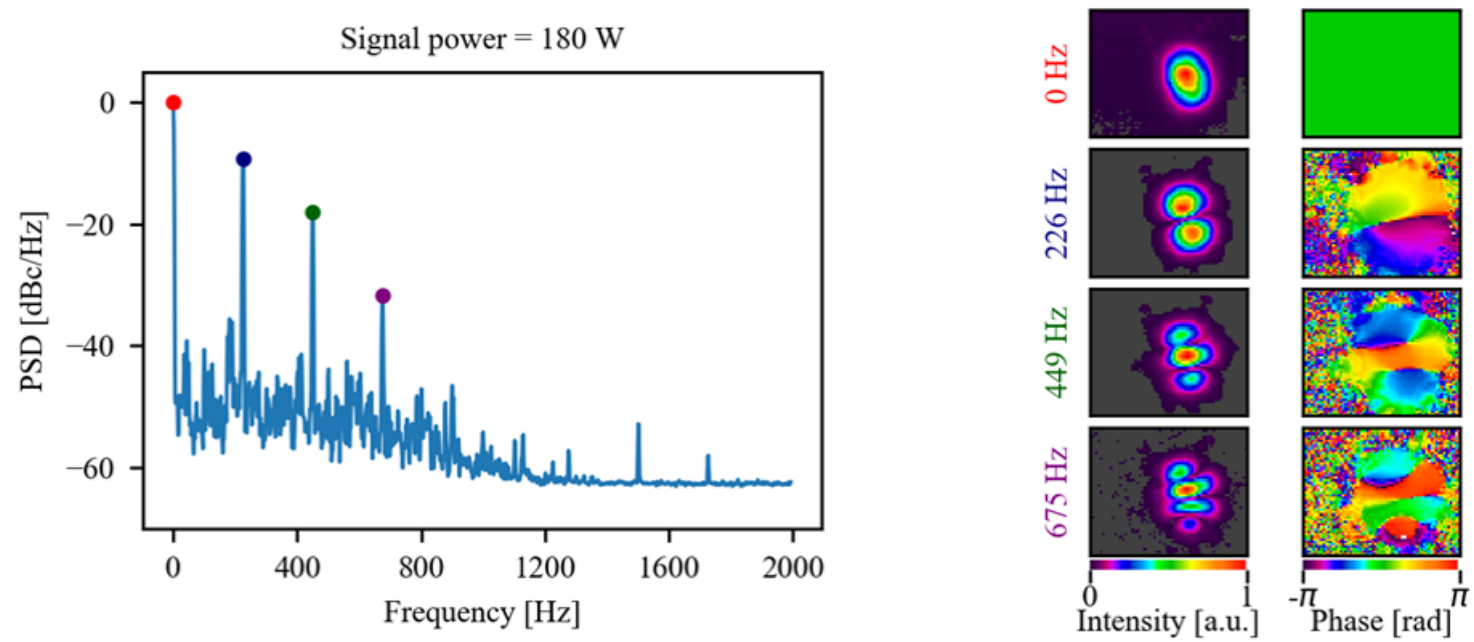

Figure 5: ST measurement of the amplifier system at $180 \mathrm{~W}$ of signal power with induced instability at 225 Hz. THe right plot shows the ST PSD while the right plots show the spatial distribution of fluctuations at four different frequencies.

where $\sigma_{P F}$ is the RMS value at the PF, $\nu_{p}$ is the PF, $\Delta \nu$ is the frequency width, and $\tilde{P}(\nu)$ is the ST PSD as a function of frequency. We have used a frequency width of $10 \mathrm{~Hz}$ for all RMS values. In Fig. 6 a) the RMS of the main TMI peak is measured as a function of PF and the frequency response from the input excitation is also included. This plot basically shows the gain of the excitation due to TMI and can almost directly be compared to simulations. ${ }^{4,6}$ The strange behaviour in the output RMS from $400 \mathrm{~Hz}$ to 500 stems from the strength of the input perturbation at these frequencies. The right plot displays the RMS peak as a function of signal power and
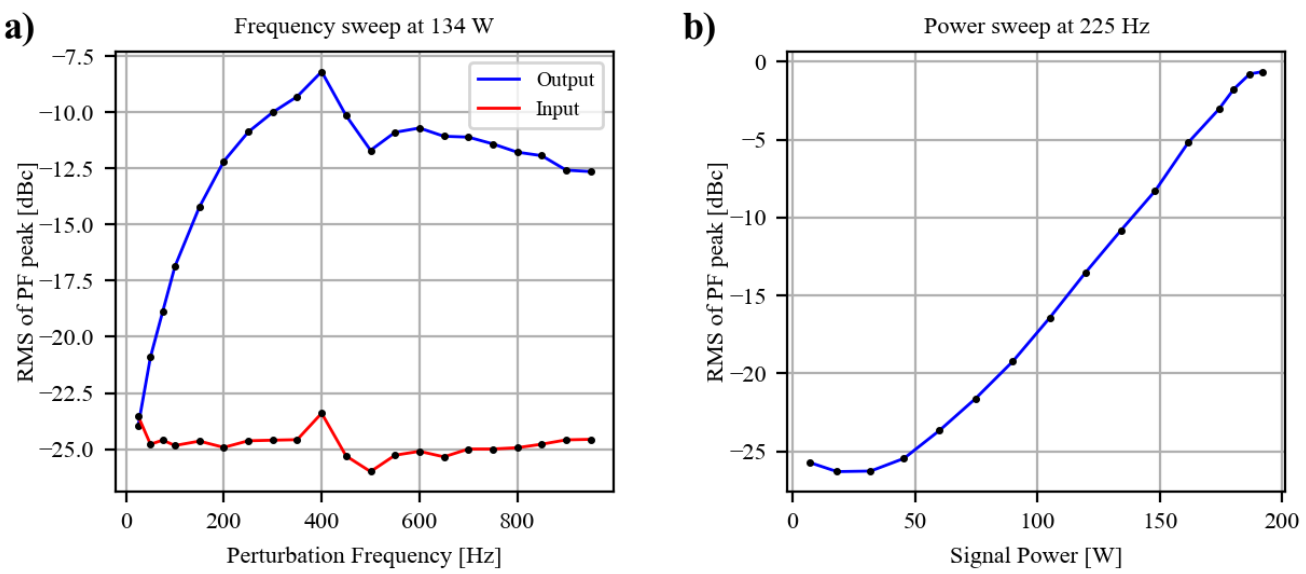

Figure 6: a) RMS of the PSD at the PF as a function of PF at a signal power of $134 \mathrm{~W}$. b) RMS of the PSD at the $\mathrm{PF}$ as a function of signal power for a $\mathrm{PF}$ of $225 \mathrm{~Hz}$.

also shows an interesting behaviour. For this measurement the exact level of input amplitude is not important as the exact same perturbation is induced for signal powers. The first point in this series is not amplified as no pump is coupled into the rod which explains decrease in RMS value to the second point. For these small signal powers the larger overlap of the $\mathrm{LP}_{01}$-mode leads to relative suppression of the $\mathrm{LP}_{11}$ power compared to the $\mathrm{LP}_{01}$ power and consequently the RMS value. The gain of the $\mathrm{LP}_{11}$-mode due to TMI however quickly 
surpasses the relative suppression due to the overlap difference even for signal powers below $50 \mathrm{~W}$. Above $50 \mathrm{~W}$ of signal power, the RMS increases exponentially until around $185 \mathrm{~W}$ where the chaotic region of TMI begins and the RMS growth at the PF stagnates.

In our previous work, ${ }^{10}$ we discussed why the higher order fluctuations from the purposefully seeded TMI did not occur in unseeded TMI. Here we presumed that the inherent noise that usually seeds TMI is broad in frequency and any higher order fluctuations will be drowned in a main fluctuation at that frequency. Higher order TMI fluctuations without purposefully seeded TMI have nonetheless been observed and the ST measurement displaying this behaviour can be seen in Fig. 7. Here the main TMI peak is at $426 \mathrm{~Hz}$ and broader than the seeded TMI from Fig. 5. This rather strong specific seeding probably comes from vibrations in the setup which can originate from a number of different sources. The second harmonic at $885 \mathrm{~Hz}$ and the third harmonic at

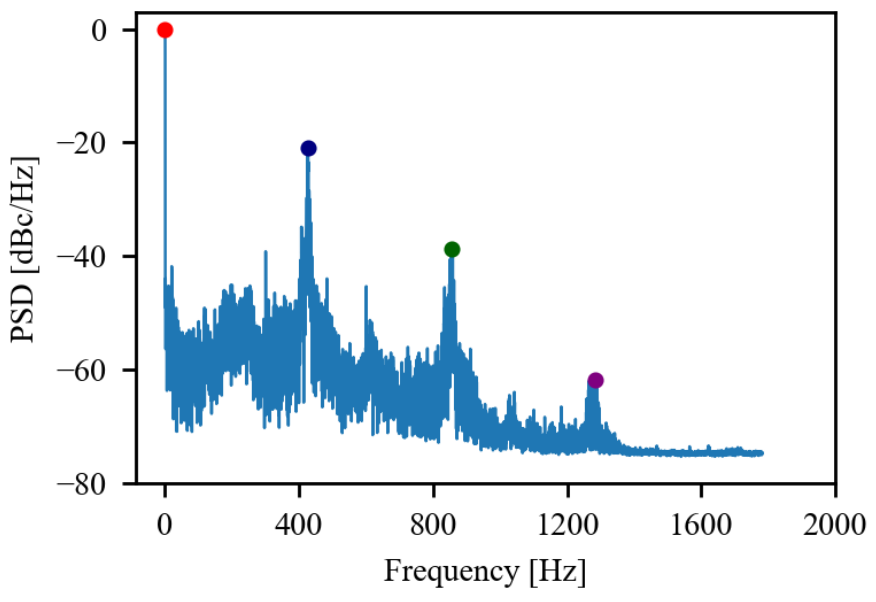

Figure 7: ASD

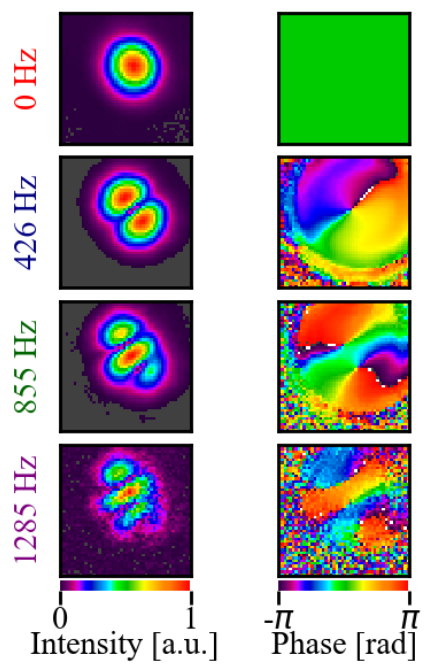

$1285 \mathrm{~Hz}$ with three and four lobes, respectively, show the exact same spatial distributions as the seeded TMI. Observing these higher-order harmonics without intentional seeding shows that all TMI peaks most likely has these harmonics but are usually dominated by a main TMI fluctuation peak. These measurement show that TMI threshold of fiber amplifiers could be increased by limiting vibrations in the amplifier setup.

\section{CONCLUSION}

In this work, an investigation of the seeding mechanism of TMI in rod fiber amplifiers using ST measurements is performed. We show that significant peaks in TMI spectra overlap with pump intensity noise which shows that pump intensity noise can directly amplify TMI fluctuations. ST measurements show that different frequencies have different orientations of $\mathrm{LP}_{11}$-mode interaction which suggests that the fundamental seeding of TMI is chaotic in nature. This notion is supported as we achieve successful seeding of TMI using a dynamic input coupling into a rod fiber amplifier. The seeded TMI is used to measure the TMI fluctuation strength as a function of both frequency and signal power. ST measurements of the seeded TMI show higher harmonics of fluctuation which are not usually observed for unintentionally seeded TMI. TMI spectra are wide and higher harmonics are dominated by main fluctuations at these frequencies. We nonetheless present a unique ST measurement where higher harmonics are observed without intentional seeding.

\section{REFERENCES}

[1] Jauregui, C., Stihler, C., and Limpert, J., "Transverse mode instability," Adv. Opt. Photon. 12, 429-484 (Jun 2020). 
[2] Eidam, T., Wirth, C., Jauregui, C., Stutzki, F., Jansen, F., Otto, H.-J., Schmidt, O., Schreiber, T., Limpert, J., and Tünnermann, A., "Experimental observations of the threshold-like onset of mode instabilities in high power fiber amplifiers," Opt. Express 19, 13218-13224 (Jul 2011).

[3] Smith, A. V. and Smith, J. J., "Mode instability in high power fiber amplifiers," Opt. Express 19, 1018010192 (May 2011).

[4] Dong, L., "Stimulated thermal rayleigh scattering in optical fibers," Opt. Express 21, 2642-2656 (Feb 2013).

[5] Jauregui, C., Eidam, T., Otto, H.-J., Stutzki, F., Jansen, F., Limpert, J., and Tünnermann, A., "Temperature-induced index gratings and their impact on mode instabilities in high-power fiber laser systems," Opt. Express 20, 440-451 (Jan 2012).

[6] Hansen, K. R., Alkeskjold, T. T., Broeng, J., and Lægsgaard, J., "Theoretical analysis of mode instability in high-power fiber amplifiers," Opt. Express 21, 1944-1971 (Jan 2013).

[7] Ward, B. G., "Modeling of transient modal instability in fiber amplifiers," Opt. Express 21, 12053-12067 (May 2013).

[8] Otto, H.-J., Stutzki, F., Jansen, F., Eidam, T., Jauregui, C., Limpert, J., and Tünnermann, A., "Temporal dynamics of mode instabilities in high-power fiber lasers and amplifiers," Opt. Express 20, 15710-15722 (Jul 2012).

[9] Christensen, S. L., Johansen, M. M., Michieletto, M., Triches, M., Huot, L., Maack, M. D., and Lægsgaard, J., "Novel high-speed camera analysis of transverse mode instabilities in rod fiber amplifiers," in [Fiber Lasers XVII: Technology and Systems], Dong, L., ed., 11260, 148 - 155, International Society for Optics and Photonics, SPIE (2020).

[10] Christensen, S. L., Johansen, M. M., Michieletto, M., Triches, M., Maack, M. D., and Lægsgaard, J., "Experimental investigations of seeding mechanisms of tmi in rod fiber amplifier using spatially and temporally resolved imaging," Opt. Express 28, 26690-26705 (Aug 2020).

[11] Stihler, C., Jauregui, C., Tünnermann, A., and Limpert, J., "The impact of pump-power noise on transverse mode instabilities," in [Fiber Lasers XVI: Technology and Systems], Carter, A. L. and Dong, L., eds., 10897, 7 - 12, International Society for Optics and Photonics, SPIE (2019).

[12] Stihler, C., Jauregui, C., Kholaif, S. E., and Limpert, J., "Intensity noise as a driver for transverse mode instability in fiber amplifiers," PhotoniX 1(1), 8 (2020).

[13] https://www.nktphotonics.com/lasers-fibers/product/aerogain-rod-high-power-ytterbium-rod-fiber-gainmodules/ (Dec 2019).

[14] https://www.baslerweb.com/en/products/cameras/area-scan-cameras/ace/aca640-750um/ (Dec 2019).

[15] Johansen, M. M., Laurila, M., Maack, M. D., Noordegraaf, D., Jakobsen, C., Alkeskjold, T. T., and Lægsgaard, J., "Frequency resolved transverse mode instability in rod fiber amplifiers," Opt. Express 21, 21847-21856 (Sep 2013).

[16] https://www.thorlabs.com/thorproduct.cfm?partnumber=POLARIS-K1S2P (April 2020). 